\title{
Fuel pump promos
}

Marlboro and other cigarette brands have found a new meaning for the phrase "pump priming money". The cigarette advertisements

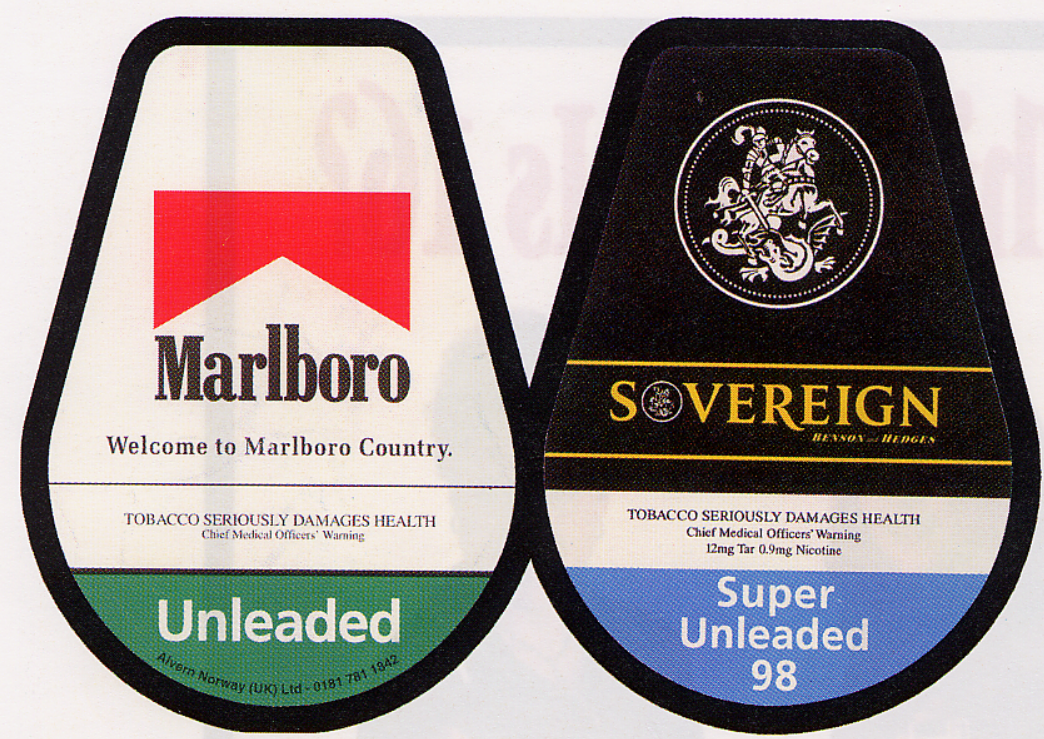

Figure 1 Fuel pump advertisements for Marlboro and Sovereign cigarettes. (Photo credit: fudith Cohen.)

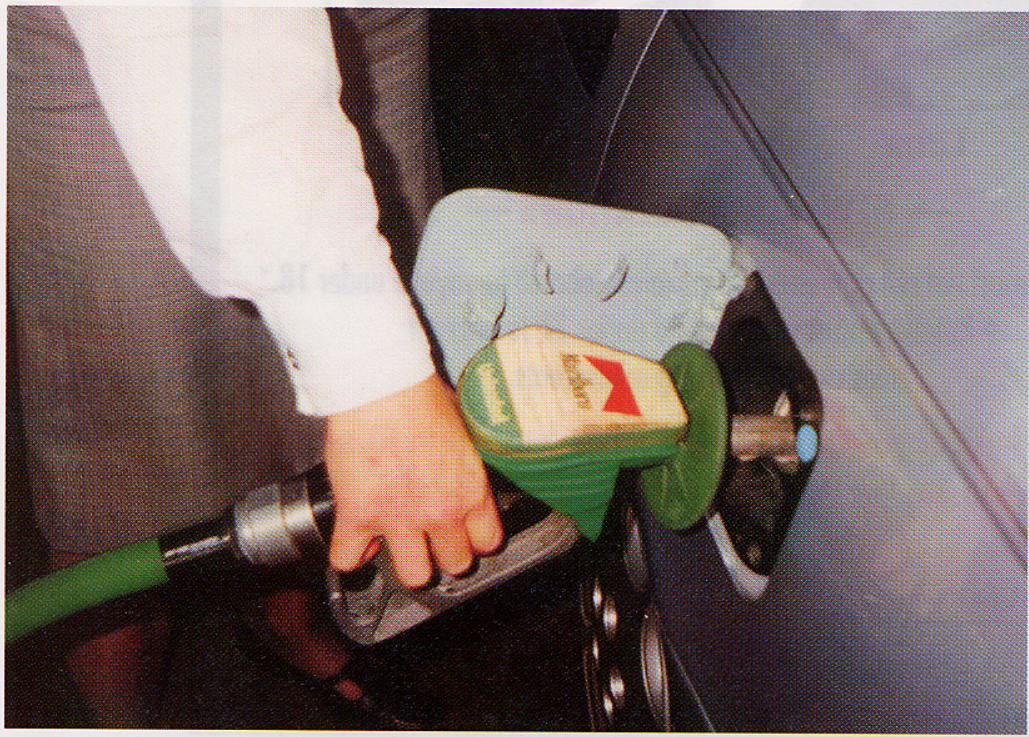

Figure 2 A Marlboro advertisement affixed to a fuel pump. (Photo credit: Fudith Cohen.) shown in the figures were at a Fina petrol (gasoline) filling station in Nottingham, England in June 1997. This form of marketing has high reach: Alvern-Norway (UK) Ltd estimates that about two million people see an advertisement on one of their nozzles each day. It is also potentially a very effective site for advertising, as their own literature spells out.

"Our advertisers are communicating their message on a one-to-one basis directly to their end-customer, in an undisturbed environment for an average of two to three minutes at a time when the customer has little alternative but to hold the message in their hand and gaze at it. In many cases, there is an opportunity to react or purchase just thirty seconds away, in the forecourt shop." (Alvern-Norway, written communication, 7 July 1997) [The forecourt is the front area of the filling station where the fuefo pumps stand.]

This marketing company hit on the idea of selling advertising space on the nozzles of fuel $\frac{0}{0}$ pumps five years ago. They now operate in 16 countries worldwide. Alvern-Norway has agreements with 22 oil companies and is currently negotiating with BP, Shell, and Mobil. There are 5500 sites carrying advertis- -0 ing in the United Kingdom: they expect coverage to reach 9000 by the end of 1997. Recognising the number of potential advertising sites, it has now registered as Alvern Forecourt Media Ltd.

The lowest total awareness figures obtained by independent research commissioned by Alvern to assess the impact of their forecourt campaigns were $50 \%$. Total awareness in Norway was $81 \%$. Spontaneous awareness of advertising for the Texas National Lottery in the USA was $31 \%$ whereas total awareness reached $71 \%$. Alvern states that its own research shows that forecourt advertising can improve brand awareness, generate an increase in sales, and improve the distribution of a product. Their material concludes: "Advertising in a forecourt environment provides an opportunity to advertise to a captive market and specifically to a mature audience. As the customer holds the advertising message for 2-3 minutes, the opportunity to see the advertising message is immense." 
Alvern Forecourt Media equip the sites, provide and maintain the units installed on the nozzles, and secure and organise the advertising. The oil companies provide the pump nozzles and approve the artwork. The funds are split equally between them. Alvern also arranges counter space for leaflets, competition entry boxes, in-store floor graphics, and printing on the reverse of till receipts and debit/credit card vouchers.

Costs depend on the number of sites, the oil company, and the location. Prices include production and printing costs but are for a minimum of 175 sites. Motorway filling stations, which have an average of 42 nozzles and a higher than average throughput, are the most expensive at $£ 250(\$ 375)$ per site per month. Asda supermarket costs $£ 230$ (\$345) per site per month but all 125 sites must be included. Esso and Texaco garages, with 20 nozzles per site, cost $£ 60$ (\$90) per site per month. Other companies, with an average of 17 nozzles, cost $£ 50(\$ 75)$ per site per month.
The only good news is that it is illegal in the United Kingdom for children aged under 16 to use the fuel pumps (a law which is adhered to), although the back seats of cars are usually level with the pumps and young children love watching the car being filled.

Twenty-nine customers are listed in the company's promotional material as having booked space and run campaigns on their medium in the past 12 months, including Gallaher Tobacco and Rothmans (which markets Marlboro and Raffles in the United Kingdom). Ironically, British filling stations all carry (small) signs by each pump that smoking is prohibited on the forecourt.

JENNIFER MINDELL

Department of Epidemiology E Public Health Imperial College School of Medicine at St. Mary's Norfolk Place, London W2 1PG, UK; email:j.mindell@ic.ac.uk

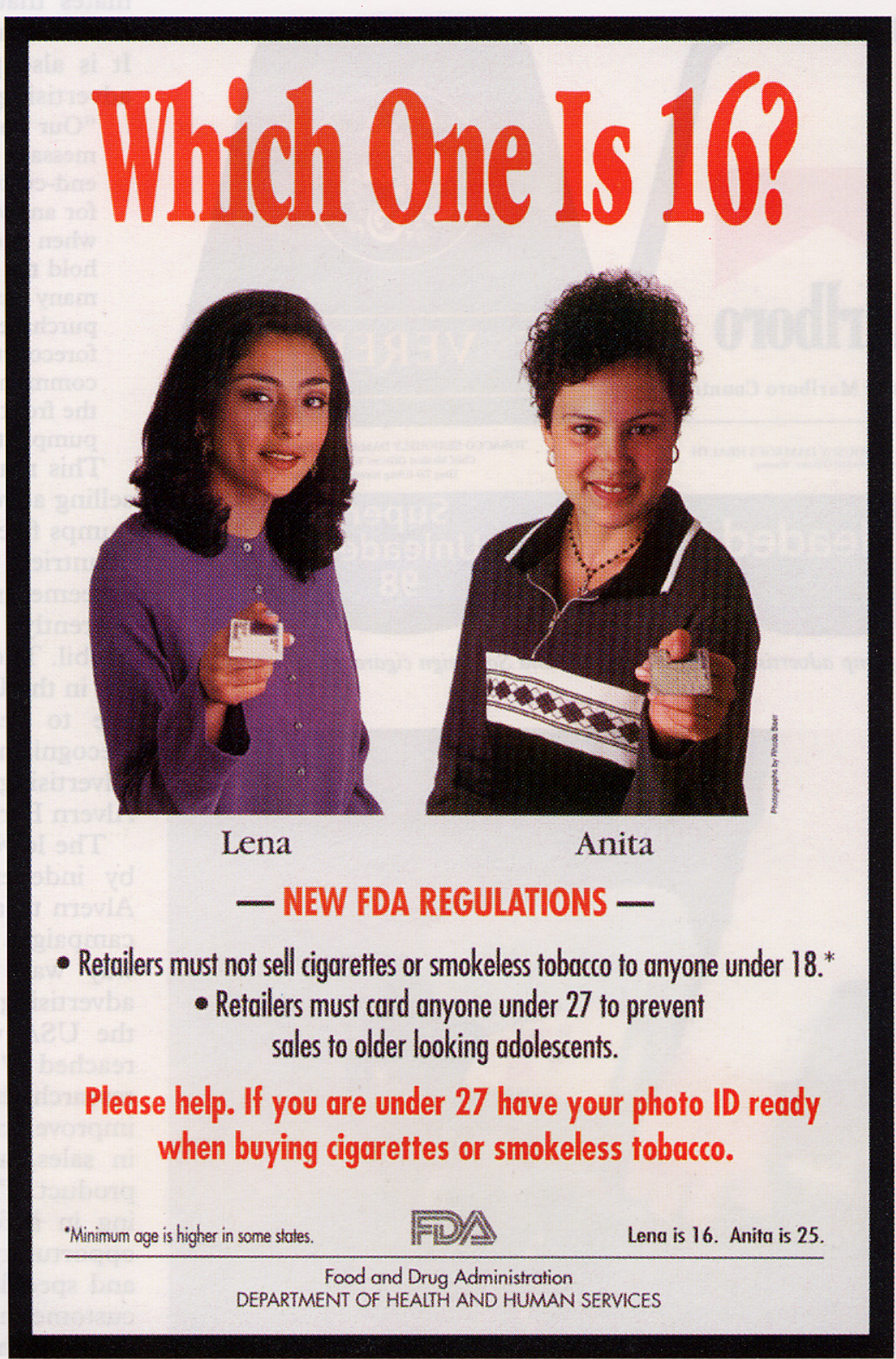

